\section{MOTHER-TO-CHILD TRANSMISSION OF KPC CARBAPENEMASE-PRODUCING KLEBSIELLA PNEUMONIAE AT BIRTH}

\author{
Paolo Bonfanti, MD, * Roberto Bellù, MD, $\uparrow$ \\ Luigi Principe, MS, $\neq$ Ilaria Caramma, MD, * \\ Manuela Condò, MD, † Tommaso Giani, MS, $\S$ \\ Gian Maria Rossolini, MD, $\$ \mathbb{\|} \| * *$ \\ and Francesco Luzzaro, MD
}

Abstract: We report on a mother-to-child transmission of KPC carbapenemase-producing Klebsiella pneumoniae at birth followed by subsequent cases in the neonatal intensive care unit. Molecular analysis of isolates showed production of KPC-3 enzyme. The only potential risk factor identified for the mother was previous activity as a caregiver. Present findings suggest consideration of proactive surveillance in pregnant women with risk factors for colonization.

Key Words: extremely low birth weight, carbapenemases, surveillance, newborns, intensive care unit

Accepted for publication June 29, 2016.

From the *Infectious Diseases Unit, $\uparrow$ Neonatal Intensive Care Unit, and $\ddagger$ Microbiology and Virology, A. Manzoni Hospital, Lecco, Italy; §Department of Medical Biotechnologies, University of Siena, Siena, Italy; and $\uparrow$ Department of Experimental and Clinical Medicine, University of Florence, \|Clinical Microbiology, Virology and Serology Unit, Florence Careggi University Hospital, and **Don Carlo Gnocchi Foundation, Florence, Italy.

The authors have no funding or conflicts of interest to disclose.

Address for correspondence: Paolo Bonfanti, MD, Infectious Diseases Unit, ASST di Lecco - Alessandro Manzoni Hospital, Via dell'Eremo, 9/11, 23900, Lecco, Italy. E-mail: pa.bonfanti@asst-lecco.it.

Supplemental digital content is available for this article. Direct URL citations appear in the printed text and are provided in the HTML and PDF versions of this article on the journal's website (www.pidj.com).

Copyright (C) 2016 Wolters Kluwer Health, Inc. All rights reserved.

DOI: $10.1097 /$ INF.0000000000001403

$\mathrm{T}^{\mathrm{h}}$ he recent dissemination of carbapenem-resistant Klebsiella pneumoniae (CR-Kp) is a major public health challenge, because of the limited treatment options and the remarkable potential for dissemination of CR-Kp in healthcare settings. ${ }^{1}$ Carbapenemase production is the most important mechanism of resistance in CR-Kp, with a number of different types of enzymes involved (e.g., KPC, IMP, VIM, NDM and OXA-48). ${ }^{2}$ KPC-type carbapenemases are among the most widespread, and KPC carbapenemase-producing $K$. pneumoniae (KPC-Kp) lineages, such as those of clonal complex 258, have caused large outbreaks and spread epidemically in several countries. ${ }^{1}$ Italy is one of these countries, where the rates of CR-Kp causing invasive infections increased from $0.9 \%$ in 2009 to $32.9 \%$ in $2014,{ }^{3}$ mostly because of a countrywide dissemination of KPC-Kp of clonal complex $258 .{ }^{4}$

Infections caused by KPC-Kp are typically healthcare-associated. Most of these infections are reported in adult and elderly patients, but pediatric patients can also be involved after exposure to healthcare practices. ${ }^{5,6}$ Here we report the first case of KPCKp infection occurring in an extremely low birth weight (ELBW) infant after perinatal transmission of the resistant pathogen, and 2 subsequent cases in the neonatal intensive care unit (NICU).

\section{CASE REPORT}

On April 2014, a 30-year-old woman, 22 weeks pregnant, was admitted to the A. Manzoni Hospital of Lecco (northern Italy) for a preterm premature rupture of membranes. The woman was citizen of Sri Lanka, but had been living in Italy since several years and had been working as a private caregiver. No recent travel to Sri Lanka was referred. The clinical conditions were good, with no fever or signs of sepsis. Ampicillin ( 2 g every 6 hours) and clarithromycin ( $500 \mathrm{mg}$ every 12 hours) were given for 6 days, in accordance with guidelines for the management of women with preterm premature rupture of membranes. ${ }^{7}$ In the $23 \mathrm{rd}$ week of pregnancy, vaginal delivery was performed because labor had started. The infant was an ELBW male of $540 \mathrm{~g}$; the APGAR score was 5 at 1 minute after birth. An endotracheal tube was inserted, assisted ventilation was started, and the infant was admitted to the hospital's NICU. Diagnosis of respiratory distress syndrome was made, based on a chest radiograph. Routine microbiologic screening for preterm infants, including throat, and auricular swabs, yielded a $\mathrm{CR}-\mathrm{Kp}$ isolate from the upper airways. Routine culture of the placenta specimen collected at birth was also positive for a CR-Kp. A rectal swab subsequently obtained from the mother showed that she was colonized by CR-Kp. The CR-Kp isolates were resistant to all tested antibiotics with the exception of gentamicin, and colistin, according to EUCAST criteria (see Table 1 for minimal inhibitory concentration values). Molecular testing by GeneXpert instrument (Xpert Carba-R, Cepheid, Sunnivale, CA) was positive for the KPC carbapenemase gene. Eight days after delivery, the newborn showed laboratory signs of sepsis including leukocytosis $(37,200 / \mu \mathrm{L})$ and platelet count decrease $(62,000 / \mu \mathrm{L})$. CR-Kp with the same resistance profile and KPC positivity by molecular testing were isolated from blood and the bronchial aspirate. Treatment with meropenem $(20 \mathrm{mg} / \mathrm{kg}$ every 8 hours $)$ plus colistin $(2.5 \mathrm{mg} / \mathrm{kg}$ twice daily) was given for 10 days. The antibiotic regimen was well-tolerated and clinical conditions rapidly improved.

Twenty-four days after delivery, the newborn developed a new episode of sepsis caused by Serratia marcescens and Enterococcus faecalis showing wild-type antimicrobial susceptibility patterns. This new episode was successfully treated with cefotaxime ( $50 \mathrm{mg} / \mathrm{kg}$ every 8 hours), gentamicin $(2.5 \mathrm{mg} / \mathrm{kg}$ every 24 hours $)$ and ampicillin ( $50 \mathrm{mg} / \mathrm{kg}$ every 6 hours) administered for 10 days.

During subsequent hospital stay, KPC-Kp was persistently detected in surveillance rectal swabs, but not from other sites. The child was discharged after 115 days, in good clinical conditions $(2590 \mathrm{~g})$. A rectal swab, performed 1 year after discharge, as a part of the follow-up program, was negative for CR-Kp.

Further molecular analysis of the CR-Kp isolates, carried out as described previously, ${ }^{4}$ revealed that they produced a KPC-3 enzyme, exhibited identical pulsed-field gel electrophoresis profiles after macrorestriction analysis with XbaI, and belonged in sequence type 512. No other carbapenemase genes were detected (including those encoding VIM-type, IMP-type, NDM-type and OXA-48-type enzymes), nor ESBL genes encoding TEM-type, SHV-type and CTX-M-type enzymes.

Until this case, no CR-Kp or other carbapenem-resistant enterics had been isolated from NICU patients of this hospital. After detection of the first CR-Kp isolate, this newborn was immediately isolated and a separate staff was dedicated to his care. Surveillance rectal swabs were obtained at admission and on a weekly basis from all infants admitted to the NICU. Forty-five days after admission of the index patient, 2 other infants were found to be colonized by CR-Kp showing identical features and pulsed-field gel electrophoresis profiles (Fig., Supplemental Digital Content 1, http://links.lww.com/INF/C595). Both infants had a low birth weight and remained colonized at the intestinal level without developing infections. A dedicated infection control team was established for investigating the outbreak and instituting additional precautionary control measures. Several meetings (commonly on weekly basis) were hold to provide an adequate response and to 
TABLE 1. Susceptibility Profile of KPC-producing $K$. pneumoniae Isolates

\begin{tabular}{lcc}
\hline \hline Antimicrobial Agent & MIC $(\mathrm{mg} / \mathrm{L})^{*}$ & Interpretation $\dagger$ \\
\hline Ampicillin & $>16$ & $\mathrm{R}$ \\
Amoxicillin-clavulanate & $>16$ & $\mathrm{R}$ \\
Piperacillin & $>128$ & $\mathrm{R}$ \\
Piperacillin-tazobactam & $>128$ & $\mathrm{R}$ \\
Cefotaxime & $>32$ & $\mathrm{R}$ \\
Ceftazidime & $>32$ & $\mathrm{R}$ \\
Cefepime & $>32$ & $\mathrm{R}$ \\
Ertapenem & $>4$ & $\mathrm{R}$ \\
Imipenem & $>8$ & $\mathrm{R}$ \\
Meropenem & $>8$ & $\mathrm{R}$ \\
Ciprofloxacin & $>2$ & $\mathrm{R}$ \\
Gentamicin & $\leq 1$ & $\mathrm{~S}$ \\
Amikacin & 16 & $\mathrm{I}$ \\
Colistin & $\leq 0.5$ & $\mathrm{~S}$ \\
Trimethoprim-sulfamethoxazole & $>8 / 152$ & $\mathrm{R}$ \\
\hline
\end{tabular}

*MIC values as measured by the Vitek system (bioMérieux, Marcy l'Etoile, France). $\dagger$ Interpretation based on current EUCAST criteria (http://www.eucast.org). MIC indicates minimal inhibitory concentration.

monitor the epidemiologic situation. Strict infection control measures were adopted. First of all, colonized neonates were placed in isolation in a separate box, with a higher spacing between cots, and managed with dedicated nursing staff ( 2 nurses for 3 infants). Other measures such as improvement of hand hygiene compliance and deep cleaning of environment and equipment were adopted. At the same time, following the consequent reduction of available cots, a temporary restriction of admissions to the NICU was decided, communicated across the neonatal regional network and applied for about 2 months. High-risk pregnant women were redirected to other regional hospitals of the network. No additional cases of CR-Kp or other carbapenem-resistant enterics were thereafter detected in the NICU.

\section{DISCUSSION}

The paper describes a case of mother-to-child transmission at birth in a ELBW infant, followed by a KPC-Kp bloodstream infection and a subsequent transmission to other 2 NICU patients. The route of transmission was supported by the timing of culture positivity, by the fact that the mother was found to be colonized by the same strain, and by the absence of circulating KPC-Kp in the NICU before admission of the index case.

This way of transmission is a well-known cause of newborn infection with several pathogens, including Streptococcus agalactiae and Enterobacteriaceae. S. agalactiae, a common gut inhabitant, plays a pivotal role as a cause of neonatal bacterial meningitis, requiring the routine implementation of a screening rectal swab in the last month of pregnancy and, in case of maternal colonization, an appropriate antimicrobial prophylaxis at birth. ${ }^{8}$ Furthermore, in the case of maternal colonization with ESBL-producing enterobacteria (that are challenging because of their multidrug resistance phenotypes), mother-to-child transmission has been demonstrated to be an important risk factor for colonization of very low birth weight infants, leading to potential epidemics in NICUs. As a consequence, routine screening of neonates and mothers has been proposed as a means of reducing neonatal morbidity and mortality. ${ }^{9}$ Similarly, present findings would suggest to consider, in endemic areas, a proactive surveillance for carbapenem-resistant enterics colonization in pregnant women with risk factors for colonization. In our case, the epidemiologic analysis showed, as the only potential risk factor, that the woman had worked since some years as a caregiver. No other risk factors (e.g., previous hospitalization in the last 2 years, prolonged use of broad-spectrum antibiotics, clinical conditions affecting the immune system) were found.

The presence of a newborn positive for a KPC-Kp strain and the subsequent outbreak posed a multifaceted challenge to the healthcare system. With regard to infection control issues, outbreaks of CR-Kp in NICUs could become an emerging problem in settings of high endemicity. ${ }^{10}$ The implementation of strict infection control measures enabled us to limit the outbreak, yet the management of the epidemic required a huge effort in terms of time, direct and indirect costs, and jeopardized the functioning of the neonatal regional network.

From a clinical point of view, little is known about the outcome of sepsis caused by CR-Kp in infants, particularly with low birth weight. Treatment of bloodstream infections in newborns is still a challenge. Symptoms of sepsis in preterm neonates are often difficult to recognize. Nevertheless, antibiotic therapy should be promptly administered, and should be appropriate for the pathogen and safe for the newborn. In our case, early administration of a colistin-based regimen was able to cure the infection, but it probably contributed to the onset of the subsequent sepsis by $S$. marcescens, a microorganism intrinsically not susceptible to polymyxins. Concerns about intravenous colistin use in adults are related to its high toxicity. Data regarding its usage in neonates with ELBW are very limited. In the case we report, colistin treatment was effective and well-tolerated.

To our best knowledge, this is the first description of a mother-to-child transmission of KPC-Kp at birth. Based on our experience, in countries with high CR-Kp endemicity, proactive surveillance procedures could be considered in pregnant women having risk factors for $\mathrm{CR}-\mathrm{Kp}$ colonization.

\section{REFERENCES}

1. Munoz-Price LS, Poirel L, Bonomo RA, et al. Clinical epidemiology of the global expansion of Klebsiella pneumoniae carbapenemases. Lancet Infect Dis. 2013;13:785-796.

2. Viau R, Frank KM, Jacobs MR, et al. Intestinal carriage of carbapenemaseproducing organisms: current status of surveillance methods. Clin Microbiol Rev. 2016;29:1-27.

3. European Centre for Disease Prevention and Control. Antimicrobial resistance surveillance in Europe 2014. Annual Report of the European Antimicrobial Resistance Surveillance Network (EARS-Net). Stockholm: ECDC; 2015.

4. Giani T, Pini B, Arena F, et al. Epidemic diffusion of KPC carbapenemaseproducing Klebsiella pneumoniae in Italy: results of the first countrywide survey, 15 May to 30 June 2011. Eurosurveill 2013;18(22):pii=20489.

5. Logan LK, Renschler JP, Gandra S, et al.; Centers for Disease Control; Prevention Epicenters Program. Carbapenem-resistant Enterobacteriaceae in children, United States, 1999-2012. Emerg Infect Dis. 2015;21: 2014-2021.

6. Yaffee AQ, Roser L, Daniels K, et al. Notes from the field: Verona integron-encoded metallo-beta-lactamase-producing carbapenem-resistant Enterobacteriaceae in a neonatal and adult intensive care unit-Kentucky, 2015. MMWR Morb Mortal Wkly Rep. 2016;65:190.

7. Yudin MH, van Schalkwyk J, Van Eyk N, et al.; Society of Obstetricians and Gynaecologists of Canada. Antibiotic therapy in preterm premature rupture of the membranes. J Obstet Gynaecol Can. 2009;31:863-7, 868.

8. Verani JR, McGee L, Schrag SJ; Division of Bacterial Diseases, National Center for Immunization and Respiratory Diseases, Centers for Disease Control and Prevention (CDC). Prevention of perinatal group B streptococcal disease-revised guidelines from CDC, 2010. MMWR Recomm Rep. 2010;59(RR-10):1-36.

9. Denkel LA, Schwab F, Kola A, et al. The mother as most important risk factor for colonization of very low birth weight (VLBW) infants with extended-spectrum $\beta$-lactamase-producing Enterobacteriaceae (ESBL-E). $J$ Antimicrob Chemother. 2014;69:2230-2237.

10. Giuffrè M, Bonura C, Geraci DM, et al. Successful control of an outbreak of colonization by Klebsiella pneumoniae carbapenemase-producing K. pneumoniae sequence type 258 in a neonatal intensive care unit, Italy. $J$ Hosp Infect. 2013;85:233-236. 